\title{
Medical treatment (excluding CPAP and orthodontics) of Obstructive Sleep Apnea/Hypopnea Syndrome (OSAHS)
}

\author{
N. Beydon', G. Aubertin², P. Garrec ${ }^{3}$ \\ 1 Pediatric pneumologist, Respiratory and Sleep Functional Exploration Unit, \\ Armand Trousseau Hospital, Paris, France \\ 2 Pediatric pneumologist, Pediatric Pneumology Dept, Rare Childhood Respiratory Diseases \\ Reference Center, Armand Trousseau Hospital, Paris, France \\ 3 MCU-PH, DCD, SQODF, UFR d'Odontologie, Paris-Diderot University, Functional Orthodontics- \\ Odontology Unit, Pitié-Salpêtrière Hospital, Paris, France; Postgraduate certificate in Odonto- \\ Stomatologic Management of Obstructive Sleep Apnea Syndrome
}

\begin{abstract}
Aside surgical and orthodontic treatment and nocturnal ventilation, there are two types of treatment adapted for childhood obstructive sleep apnea-hypopnea syndrome (OSAHS): pharmacological treatment is reserved to non-severe OSAHS with no upper airway obstruction, because of limited efficacy; oro-myofacial rehabilitation is always associated to other therapeutic interventions.
\end{abstract}

KEYWORDS

Obstructive sleep apnea syndrome, children, treatment

Medical management of childhood obstructive sleep apnea/hypopnea syndrome (OSAHS) differs from surgical or instrumental treatment (ENT, orthodontics, ventilation), and comprises pharmacologic treatment and oro-myofacial rehabilitation.

\section{PHARMACOLOGIC TREATMENT}

As, in adults at least, an inflammatory component is found in the genesis and persistence of OSAHS, anti-inflammatory treatments have been tested in children. Two types of molecule have been used, in isolation or association: corticosteroids, and antileukotriene agents (mainly montelukast).

\section{Local corticosteroids}

Intranasal corticosteroids were initially shown to provide benefit in the clinical symptomatology of childhood nasal obstruction ${ }^{3}$. Efficacy against OSAHS has been harder to show, probably because the 
effect is relatively light. A randomized double-blind study targeting mild OSAHS found significant reduction in apnea/hypopnea index (AHI) in 48 children treated with intranasal corticosteroids compared to placebo ${ }^{7}$. Efficacy, however, was only partial as, despite the mild form of OSAHS, only half of the children recovered normal AHI.

\section{Antileukotriene agents}

The importance of leukotrienes in adenoid and tonsillar inflammation was suggested by an increase in leukotriene concentration and in the number of adenoid and tonsillar leukotriene receptors in children with OSAHS compared to rates in lymphoid tissue in children with iterative pharyngitis ${ }^{4}$. Antileukotrienes proved effective in childhood OSAHS in a randomized double-blind placebo-controlled study of 2 groups of 23 children each ${ }^{5}$; only the antileukotriene group showed radiologic regression of adenoid hypertrophy and reduced $\mathrm{AHI}$.

\section{Drug associations}

Finally, an association of the above (antileukotrienes and intranasal corticosteroids) was tested in children with mild residual OSAHS (AHI 1-5/h) following adenotonsillectomy ${ }^{6}$. All 4 study indices (apnea, apnea/ hypopnea, micro-arousal and lowest percutaneous arterial oxygen saturation during sleep) were significantly improved in the treatment group (n $=22$ ) compared to the group without treatment $(n=14)$.

\section{Indications for medical treatment}

The above reports led to a clinical consensus in favor of trying medical treatment in mild to moderate OSAHS in the absence of serious anatomic ENT obstacle or residual obstacle after adenotonsillectomy.

\section{Precautions}

It should be clear that medical management has not been proved to have great efficacy. Also, as usual, sideeffects need to be known to assess the risk/benefit ratio.

Intranasal corticosteroids often induce local bleeding, and may be contraindicated in patients presenting with frequent or abundant epistaxis. The other tolerance issue to be monitored is onset of mycosis, the absence of which should be checked before initiating treatment. Finally, at the dosage prescribed by the AMM, growth is conserved, but should be monitored, as overdose easily occurs with this kind of presentation and can be hard to detect. Montelukast which is the only antileukotriene available in France) is reputed to have few sideeffects; epidemiological studies, however, spotlighted this molecule, which cumulated the greatest number of side-effects, especially psychiatric ${ }^{2}$, in children ${ }^{1}$. In practice, if the child shows behavioral problems (notably, anxiety or depression, or agitation) or simply nightmares, the parents should be warned of the risk of aggravation, in which case montelukast should be immediately discontinued; in such cases, the physician may equally abstain from prescribing it in the first place. 


\section{OROFACIAL MUSCULAR REHABILITATION}

Orofacial hypotony is by no means a rare finding in children with OSAHS. It is often easily diagnosed on first inspection, with labial opening at rest, anterior protrusion of the tongue at rest, and chewing/swallowing disorder. As well as treating the underlying cause (e.g., nasal obstruction), the hypotony should be entirely rehabilitated in order to allow favorable evolution of OSAHS.

The objectives of rehabilitation are: to reinforce the muscles of the tongue and face; to avoid oral breathing; to raise the position of the tongue within the mouth in sleep and when awake, so as to limit impact on dental arcade growth; and to check the size and mobility of the lingual frenum.

Practitioners (physiotherapists and speech therapists) need specific training. Rehabilitation, in the therapist's office, should at first be intensive. In the longer term, it is essential for the child to perform the exercises several times a day at home, usually accompanied by an adult. This requires considerable motivation on the part of child and family, which is easier to obtain in over-6 year-olds. At the same time, oro-myofacial rehabilitation is more effective the further the child is from puberty.

\section{CONCLUSION}

There are medical treatments and functional rehabilitation programs that may be effective in childhood OSAHS. Indications are founded on fairly consensual clinical experience.

Conflicts of interest: The authors declare no conflicts of interest.

\section{REFERENCES}

1. Brunlöf G, Tukukino C, Wallerstedt SM. Individual case safety reports in children in commonly used drug groups - signal detection. BMC Clin Pharmacol 2008;8:1.

2. Bygdell M, Brunlöf G, Wallerstedt SM, Kindblom JM. Psychiatric adverse drug reactions reported during a 10-year period in the Swedish pediatric population. Pharmacoepidemiol Drug Saf 2012;21:79-86.

3. Demain JG, Goetz DW. Pediatric adenoidal hypertrophy and nasal airway obstruction: reduction with aqueous nasal beclomethasone. Pediatrics 1995;95:355-64.

4. Goldbart AD, Goldman JL, Veling MC, Gozal D. Leukotriene modifier therapy for mild sleep-disordered breathing in children. Am J Respir Crit Care Med 2005;172:364-70. 
5. Goldbart AD, Greenberg-Dotan S, Tal A. Montelukast for children with obstructive sleep apnea: a double-blind, placebo-controlled study. Pediatrics 2012;130:e575-80.

6. Kheirandish L, Goldbart AD, Gozal D. Intranasal steroids and oral leukotriene modifier therapy in residual sleep-disordered breathing after tonsillectomy and adenoidectomy in children. Pediatrics 2006;117:e61-6.

7. Kheirandish-Gozal L, Gozal D. Intranasal budesonide treatment for children with mild obstructive sleep apnea syndrome. Pediatrics 2008;122:e149-55. 